\title{
Stem cells in the periodontal ligament
}

S. Ivanovski, S. Gronthos2, S. Shi, P. M. Bartold

\section{Abstract}

The ability to identify and manipulate stem cells has been a significant advancement in regenerative medicine and has contributed to the development of tissue engineering-based clinical therapies. Difficulties associated with achieving predictable periodontal regeneration, means that novel techniques such as tissue engineering need to be developed in order to regenerate the extensive soft and hard tissue destruction that results from periodontitis. One of the critical requirements for a tissue engineering approach is the delivery of ex vivo expanded progenitor populations or the mobilization of endogenous progenitor cells capable of proliferating and differentiating into the required tissues. By definition, stem cells fulfill these requirements and the recent identification of stem cells within the periodontal ligament represents a significant development in the progress toward predictable periodontal regeneration. In order to explore the importance of stem cells in periodontal wound healing and regeneration, this review will examine contemporary concepts in stem cell biology, the role of periodontal ligament progenitor cells in the regenerative process, recent developments in identifying periodontal stem cells and the clinical implications of these findings. Oral Diseases (2006) 12, 358-363

\section{Mesenchymal stem cells and their identification}

Stem cell is a broad term used to describe a wide variety of cells from varying sources. Stem cells can be broadly divided into two categories - embryonic and adult. Embryonic stem cells are totipotent cells, capable of differentiating into virtually any cell type, as well as being propagated indefinitely in an undifferentiated state (Evans and Kaufman, 1981; Keller, 1995). Due to regulatory issues associated with the use of embryonic stem cells, and the difficulty in controlling their growth and differentiation, recent attention has been focused on stem cells derived from adult tissues. Indeed, from a practical standpoint, adult stem cells are more appropriate for periodontal tissue engineering purposes. Although it is accepted that adult stem cells have a more restricted differentiation potential compared with the totipotent properties of embryonic stem cells, these cells still fulfill the 
basic characteristics of stem cells - abilities to self-renew, generate large numbers of progeny and differentiate into multiple mature cell types (Fortier, 2005). As adult stem cells are not totipotent, they can be further classified depending on their origin and differentiation potential. Two common examples are hematopoietic and mesenchymal stem cells (MSCs). As the critical tissues that require regeneration in the periodontium (cementum, ligament, bone) are of mesenchymal origin, it is MSCs that are required for periodontal regeneration and will be the focus of this review.

Mesenchymal stem cells, like all stem cells, share at least two characteristics (Kemp et al, 2005).

Firstly, they can give rise to mature cell types that have characteristic morphologies and specialized functions. Secondly, the cells are capable of self-renewal for the lifetime of the organism and are defined by their clonogenic potential. In other words, a single adult stem cell should be able to generate a line of genetically identical cells, which then give rise to all the appropriate, differentiated cell types of the tissue in which it resides. These self-renewal and clonogenic properties are difficult to demonstrate in vivo, but can be implied by showing that a stem cell is clonogenic in vitro, or that a purified population of candidate stem cells can regenerate the native tissue and be subsequently isolated from this regenerated tissue (Evans and Potten, 1991; Gronthos et al, 2002).

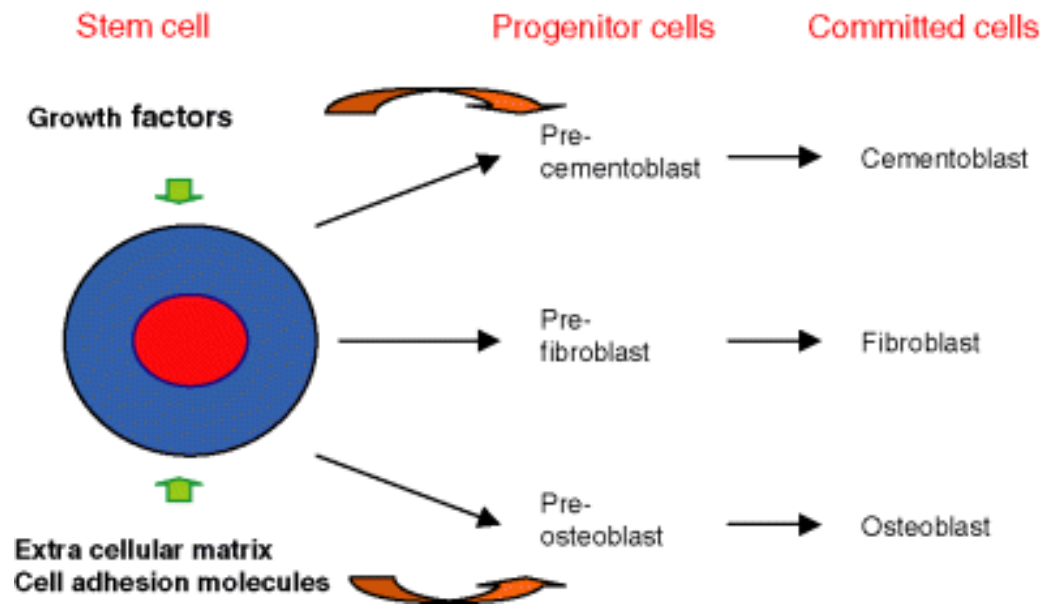

Figure 1. Differentiation of adult mesenchymal stem cells and progenitor cells into periodontal cells 
Typically, stem cells generate intermediate cell types before they achieve their fully differentiated state. The intermediate cell is called a precursor or progenitor cell. Progenitor or precursor cells in adult tissues are partly differentiated cells that divide and give rise to differentiated cells (Figure 1 ). Such cells are usually regarded as 'committed' to differentiating along a particular cellular development pathway. Generally, undifferentiated cells are considered to be 'progenitor' cells until their multi-tissue differentiation and self-renewal properties can be demonstrated in order to designate them as 'stem' cells.

Unlike embryonic stem cells, which are defined by their origin (the inner cell mass of the blastocyst), adult stem cells share no such definitive means of characterization and their origin in mature tissues is unknown. As MSCs were originally identified from bone marrow stroma (Friedenstein et al, 1966), most studies aimed at isolating MSCs sought ways of separating stromal cells with a fibroblast-like morphology from hematopoietic cell populations that make up the vast majority of bone marrow biopsies. A commonly utilized technique for this type of cell sorting is flow cytometry, which utilizes a laser beam to separate cell fractions from a larger population by utilizing the differential lightabsorbing or fluorescing properties of certain cell subpopulations.

Flow cytometry studies have shown that MSC share many similar cell surface markers with mature hematopoietic cells (Majumdar et al, 2003). Thus, identification of cell surface markers that are 'specific' to MSCs is very important for their isolation. STRO-1, a mouse-raised immunoglobulin M antibody, has been shown to identify a cell surface antigen expressed by stromal cell precursors in human bone marrow. STRO-1 has been shown to be non-reactive with hematopoietic progenitors, but included within the STRO-1-positive population are all detectable clonogenic colony-forming units that morphologically resemble fibroblasts (Simmons and Torok-Storb, 1991). 
However, no single antigenic marker has been shown to be specific for MSCs and therefore, combinations of antibodies must be utilized. Other cell surface antigens such as CD146/MUC-18, pericyte-associated antigen CD106 (3G5), CD-44, VCAM-1, alkaline phosphatase and $\alpha$-smooth muscle actins have also been identified on STRO-1-positive cells (Simmons and Torok-Storb, 1991; Shih, 1999; Gronthos et al, 2000, 2002, 2003), but may also be found on other 'committed' cell lineages (Kemp et al, 2005). For example, CD146 (MUC-18/Mel-CAM), is also known to be present on smooth muscle, endothelium, myofibroblast and Schwann cells in situ, as well as being a marker for some human neoplasms (Shih, 1999). Therefore, although cell sorting using STRO-1 and CD146 has isolated cells with clonogenic properties and multi-lineage potentials, it is possible that these cells represent a stem-cell-enriched population that also contains progenitor cells (Kramer et al, 2004). Further identification of more specific markers for stem cells using molecular and genetic approaches are required (Bianco et al, 2001; Shi and Gronthos, 2003) in order to obtain 'pure' populations of stem cells.

\section{Progenitor cells involved in periodontal wound healing and regeneration}

The functional periodontal attachment apparatus anchors the tooth and consists of periodontal fibers that run between alveolar bone and cementum lining the root surface. Furthermore, gingival connective tissues and epithelium overlay the alveolar bone and form a dentogingival junction at the interface with the tooth. The complex structure of the periodontium, which consists of the soft connective tissues of the gingiva and periodontal ligament, as well as the mineralized tissues cementum and bone, makes periodontal wound healing a unique process. Following conventional periodontal therapy involving debridement of the root surface, the periodontal tissues heal by repair and the migration of the epithelium along the previously contaminated root surface (long junctional epithelium), which prevents connective tissue attachment to the root surface (Caton et al, 1980). 
Regeneration of the attachment apparatus that is destroyed as a result of periodontitis has long been the goal of periodontal therapy. Periodontal regeneration requires new connective tissue attachment to the root surface, a process that involves the regeneration of periodontal fibers and the insertion of these fibers into newly formed cementum. Unfortunately, currently available regeneration techniques are clinically unpredictable, resulting in only partial regeneration at best (Bartold et al, 2000; Wang et al, 2005; Zohar and Tenenbaum, 2005).

From a biological perspective, current and future prospects for improved regeneration of periodontal tissues are dependent on our ability to facilitate the re-population of the periodontal wound by cells capable of promoting regeneration. From this perspective, the periodontal ligament has been shown to be of critical importance in the regenerative process. It has been demonstrated that only the periodontal ligament, but not gingival connective tissue or bone, contains cells capable of establishing new attachment fibers between cementum and bone (Karring et al, 1980; Nyman et al, 1980). It has also been shown that gingival fibroblasts grown in vitro failed to contribute to reformation of periodontal ligament around teeth implanted in dogs, while periodontal ligament fibroblasts were observed to either contribute to regeneration or at least not to inhibit regeneration (Boyko et al, 1981). The ability of periodontal ligament cell populations to achieve regeneration has implied that progenitor cells, and possibly stem cells, exist within the periodontal ligament.

Although it is clear that cells residing in the periodontal ligament can achieve regeneration, this population is heterogenous (Limeback et al, 1983) and it is not known which subpopulations are capable of achieving regeneration. Indeed, cells derived from regenerating defects were found to have specific properties, such as increased proliferation rates, representative of a 'regenerative' phenotype, and distinct from periodontal ligament cells (Ivanovski et al, 2001). Therefore, in order to identify progenitor and stem cells from the periodontium, it would be useful to establish where 
these cells reside within the periodontium, as well as identify markers that can be used to distinguish these types of cells.

Periodontal regeneration during wound healing is likely to share many common biological processes with those of periodontal development. Apart from the gingival epithelium, the functional periodontium is almost entirely (with the exception of neural and vascular elements) derived from ectomesenchyme, the embryonic connective tissue derived from neuroectoderm. Mesenchymal cells are precursors for a diverse group of connective tissue cells including smooth muscle cells, endothelial cells, chondroblasts, pericytes, adipocytes, osteoblasts and fibroblasts. At the initiation of tooth formation in fetal jaws, mesenchymal cells are induced by the overlying oral epithelium to an odontogenic specificity. Initially, this interaction results in the formation of the tooth crown comprising the ectoderm (oral epithelium)-derived enamel and the mesenchyme-derived dentin. Subsequently, during root formation and the development of the periodontal attachment apparatus, epithelial root sheath cells derived from the enamel organ fragment to allow the attachment of mesenchymal cells on the exposed root dentine. These mesenchymal cells proceed to deposit cementum and establish an attachment apparatus. In order to understand the cellular origins of the developing periodontal attachment apparatus, transplanted tooth buds were used to show that the mesenchymal-derived dental follicle surrounding the developing tooth root is the source of progenitor cells for cementum, alveolar bone and periodontal ligament (Ten Cate et al, 1971; Ten Cate and Mills, 1972; Palmer and Lumsden, 1987). Further evidence that cells from the dental follicle are precursors to cementoblasts has been shown by direct cell migration in the rat molars (Cho and Garant, 1988, 1989). More recently, the dental follicle associated with third molars has been shown to contain precursor cells which are clonogenic and have the ability to differentiate under in vitro conditions to a membrane-like structure containing calcified nodules (Morsczeck et al, 2005). 
The source of post-natal progenitor cells which may be capable of regenerating the periodontium has been investigated for a number of years. Cell kinetic experiments in mice and rats (McCulloch and Melcher, 1983b; McCulloch et al, 1989) have shown that periodontal ligament fibroblast populations represent a steady-state renewal system, with the number of new cells generated by mitosis equal to the number of cells lost through apoptosis and migration (McCulloch and Melcher, 1983a). This capacity for self-renewal, which is further evidenced by the rapid turnover of the periodontal ligament, supports the notion of progenitor/stem cell populations. Furthermore, a significant number of periodontal cells do not enter the cell cycle (McCulloch and Melcher, 1983c), suggesting that these cells may act in a similar manner to quiescent, self-renewable and multipotent stem cells.

The relationship between progenitor cells in regenerating tissues and normally functioning (steadystate) tissues has been investigated in studies performed in normal mouse periodontal ligament (McCulloch, 1985), wounded mouse periodontal ligament (Gould et al, 1980), normal rat gingiva (Pender et al, 1988) and inflamed monkey gingiva (Nemeth et al, 1993). These studies have identified a common paravascular location for fibroblast progenitors. These cells exhibit some of the classical cytological features of stem cells, including small size, responsiveness to stimulating factors, and slow cycle time (Gould et al, 1980; McCulloch and Melcher, 1983a; McCulloch, 1985). Furthermore, these paravascular cells exhibit spatial clustering which suggests a possible clonal distribution of progenitors and their progeny (McCulloch, 1985). Other possible sources of osteoblast and cementoblast precursors are the endosteal spaces of alveolar bone from which cells have been observed to adopt a paravascular location in the periodontal ligament of mice (McCulloch et al, 1987). 
However, based on these studies, paravascular cells in the periodontal ligament cannot be designated as stem cells because the clonogenic capacity of these cells, and their ability to differentiate into multiple cell types, was not demonstrated. Additional identification and isolation of these cells using membrane surface markers for stem cells, and demonstration of their clonogenic as well as multi-lineage properties, has subsequently been sought in order to consolidate the hypothesis that stem cells reside within the periodontal ligament.

\section{Stem cells in the periodontal ligament}

More than any other tissue, the complexity of the periodontium along with the understanding that only certain components are capable of achieving regeneration, makes the identification of stem cells important, not only in the understanding of normal wound healing events but also in designing novel clinical techniques. The inherent properties of stem cells, including their ability to form different tissue types and self-renew, make their presence within the healing periodontal defect desirable in order to facilitate periodontal regeneration. Therefore, the ability to identify, characterize and manipulate stem cells within the periodontium is of considerable clinical significance, especially in terms of developing novel mechanisms of achieving periodontal regeneration.

Although it is well established that precursor cells exist within the periodontal ligament, stem cell properties such as self-renewal, clonogenicity and multi-tissue differentiation potential have only recently begun to be demonstrated. For these properties to be determined, it was necessary to isolate stem cells in vitro using available markers for the identification of MSCs. However, although the use of stromal cell markers and the absence of hematopoietic cell markers has been successful in isolating stem cells from bone marrow, additional challenges have been encountered when trying to 
extract MSCs from connective tissues composed largely of fibroblastic cells, such as the periodontal ligament. As stated earlier, a single specific antigenic marker for MSCs is not available, and hence a combination of markers must be used in order to achieve their isolation (Kemp et al, 2005). A methodology for isolating MSCs from the periodontal ligament has been based on techniques shown to successfully isolate bone marrow-derived MSCs and stem cells in dental pulp cells from adult and edentulous teeth (Gronthos et al, 2000, 2002, 2003; Miura et al, 2003).

Mesenchymal stem cells from post-natal human pulp express the stem cell marker STRO-1, as well as CD44, integrin $\beta 1$, VCAM-1, CD146/MUC-18 and alkaline phosphatase (Gronthos et al, 2000, 2002). When dental pulp stem cells (DPSCs) were transplanted into immunocompromised mice, they generated a dentin-like structure lined with human odontoblast-like cells that surround a pulp-like interstitial tissue. The DPSCs were also capable of multi-lineage differentiation into adipocytes and neural-like cells (Gronthos et al, 2000, 2002). Stem cells have also been isolated from the pulp of human exfoliated deciduous teeth (SHED) (Miura et al, 2003). Immunohistochemical analyses of these cells have shown that they are positive for STRO-1 and CD-146/MUC-18. After transplantation into immunocompromised mice, SHED were found to induce bone formation, generate dentin, survive in the mouse brain and express neural tissue markers. It has been suggested that SHED represent a population of multipotent stem cells that are more immature in the cell hierarchy than previously examined post-natal stem cell populations. Subsequent research using frozen pulp and bone marrow sections has indicated that MSCs are located in perivascular regions and they constitutively express STRO-1, CD146 and $\alpha$-smooth muscle actin on the cell surface (Shi and Gronthos, 2003). The perivascularlocalization of these cells is consistent with previous reports of localization of progenitor cells in the periodontal ligament. 
Using a methodology similar to that utilized to isolate MSCs from deciduous and adult pulp, multipotent post-natal stem cells from human periodontal ligament (PDLSCs) have also been described (Seo et al, 2004). Cultured cells were expanded from single cell suspensions derived from periodontal ligament tissue and the presence of stem cells was determined using antibodies such as STRO-1 and CD146. Under defined cultured conditions, PDLSCs differentiated into cementoblast-like cells, adipocytes and collagen-forming cells. Immunohistochemical staining and Western blot analysis showed that cultured PDLSCs express an array of cementoblastic/osteoblastic markers, including alkaline phosphatase, bone sialoprotein, osteocalcin and transforming growth factor- $\beta$ receptor type I. The cells also expressed scleraxis, a tendon transcription factor and under certain conditions become adipogenic.

These PDLSCs were transplanted into artificially created periodontal defects in the mandibular molars in rats. Histological evaluations 6-8 weeks after implantation showed that these cells had the capacity to generate a thin layer of cementum-like tissue on the surface of the hydroxyapatite/tricalcium phosphate ceramic particles carrier, along with condensed collagen fibers that resembled Sharpey's fibers. Thus, from the findings of this study, it appears that PDLSCs can be isolated using STRO-1 and CD146 markers. The presence of MSCs in the periodontal ligament is also supported by the findings of Trubiani et al (2005), who isolated and characterized a population of MSCs from the periodontal ligament which expressed a variety of stromal cells markers (CD90, CD29, CD44, CD166, CD105, CD13). The clinical potential for the use of periodontal ligament-derived stem cells has been further enhanced by the demonstration that these cells can be isolated from cryopreserved periodontal ligaments, thus providing a ready source of MSCs (Seo et al, 2005).

\section{Future prospects for periodontal regeneration}


Numerous clinical techniques, including bone grafts, root surface conditioning, barrier membranes and various growth factors, have been utilized over the years in an attempt to achieve periodontal regeneration. Unfortunately, current therapeutic measures are unable to achieve predictable regeneration, thus underscoring the importance of restoring or providing the cells and microenvironment capable of initiating and promoting new periodontal tissue formation.

From a biological perspective, in order for periodontal regeneration to occur, the availability of appropriate cell types, together with a favorable local environment promoting cell migration, adhesion, proliferation and differentiation, all need to be precisely coordinated both temporally and spatially. Thus, a tissue engineering strategy for periodontal regeneration that exploits the regenerative capacity of stem cells residing within the periodontium, grown in a three-dimensional construct and subsequently implanted into the defect may help to overcome many limitations with current regeneration modalities (Bartold et al, 2000) (Figure 2). In doing so, the need for recruitment of various different cells to the site is negated and the predictability of outcome may be enhanced. 


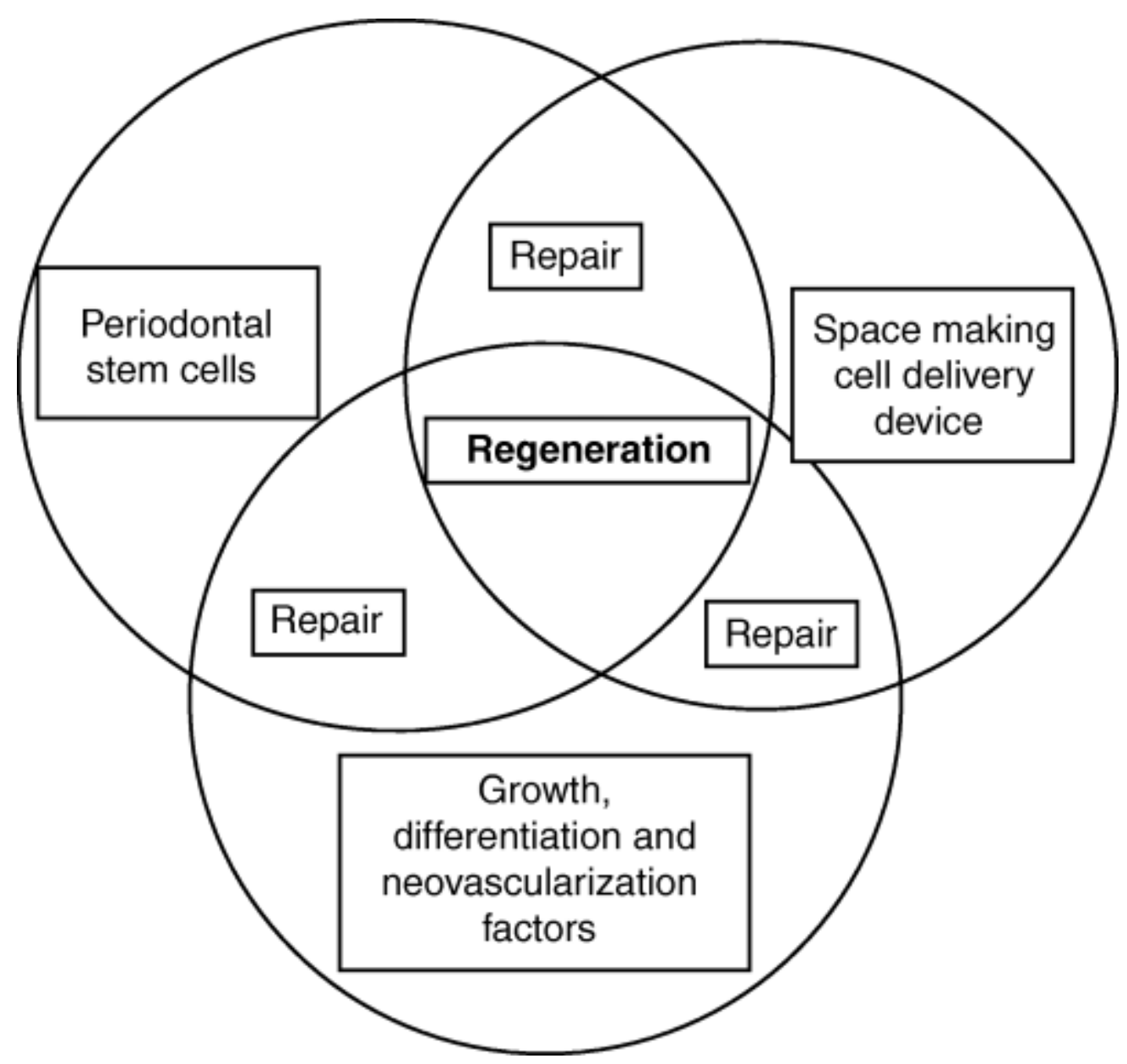

Figure 2: Schematic representation of a tissue engineering approach to periodontal regeneration using periodontal ligament stem cells. When the cells are incorporated into a suitable space making delivery device and exposed to the correct growth differentiation, neovascularization agents tissue regeneration would be expected. Should only two of the three requirements be available then repair rather than regeneration will result

Mesenchymal stem cells are already being utilized in regenerative medicine, most notably in orthopedic medicine where they have been shown to facilitate repair of bone (Mankani et al, 2001) and cartilage (Murphy et al, 2003). Furthermore, MSCs have also been shown to produce de novo myocardium (Stamm et al, 2003), as well as assist in the functional recovery following spinal cord injury (Mezey et al, 2003). The potential of stem cell-based tissue engineering has also been harnessed in the reconstruction of murine teeth using cultured stem cells which, when transferred into renal capsules, resulted in the development of tooth structures and associated bone (Ohazama et al, 2004). These results provide significant advances toward the creation of artificial embryonic 
tooth primodia from cultured stem cells that can be used to replace missing teeth following transplantation into the adult.

The plausibility of a stem cell-based tissue engineering approach to achieving periodontal regeneration is supported by animal studies demonstrating that periodontal ligament cells cultured in vitro can be successfully reimplanted into periodontal defects in order to promote periodontal regeneration (Hasegawa et al, 2005). In light of these findings, the identification of stem cells within the periodontal ligament with the ability to achieve new attachment formation in vivo is a significant development toward a tissue engineering approach to treating periodontal regeneration. Furthermore, the ready availability of periodontal ligament tissue from redundant teeth such as third molars, may provide a supply of stem cells that may be utilized for regenerating other body parts.

\section{Acknowledgements}

Original work by the authors detailed in this review was variously funded by grants from the National Health and Medical Research Council of Australia and the National Institutes of Health, Bethesda, MD, USA.

\section{References}

Bartold, PM, McCulloch, CAG, Narayanan, AS, Pitaru, S (2000). Tissue engineering: a new paradigm for periodontal regeneration base on molecular and cell biology. Periodontol 2000 24: 253269.

Bianco, P, Riminucci, M, Gronthos, S, Gehron Robey, PG ( 2001). Bone marrow stromal stem cells: nature, biology and potential applications. Stem Cells 19: 180- 192.

Boyko, G, Melcher, AH, Brunette, DM ( 1981). Formation of new periodontal ligament by periodontal ligament cells implanted in vivo after culture in vitro. J Periodontal Res 16: 73-88.

Caton, J, Nyman, S, Zander, H ( 1980). Histometric evaluation of periodontal surgery II. Connective tissue attachment levels after four regenerative procedures. J Clin Periodontol 7: 224- 231.

Cho, MI, Garant, PR ( 1988). Ultrastructure evidence of directed cell migration during initial cementoblast differentiation in root formation. J Periodontal Res 23: 268- 276. 
Cho, MI, Garant, PR ( 1989). Radioautographic study of [3H] manose ultilization during cementoblast differentiation, formation of acellular cementum, and the development of periodontal ligament principal fibers. Anat Rec 223: 209-222.

Evans, MJ, Kaufman, MH ( 1981). Establishment in culture of pluripotent cells from mouse embryos. Nature 292: 154- 156.

Evans, GS, Potten, CS ( 1991). Stem cells and the elixir of life. Bioessays 13: 135- 138.

Fortier, LA ( 2005). Stem cells: classification, controversies and clinical applications. Vet Surg 34: $415-423$.

Friedenstein, AJ, Piatetzky, S, Petrakova, KV ( 1966). Osteogenesis in transplants of bone marrow cells. J Embryol Exp Morphol 16: 381- 390.

Gould, TRL, Melcher, AH, Brunette, DM ( 1980). Migration and division of progenitor cell populations in periodontal ligament after wounding. J Periodontal Res 15: 20- 42.

Gronthos, S, Mankani, M, Brahim, J, Gehron Robey, PG, Shi, S ( 2000). Postnatal human dental pulp stem cells (DPSCs) in vitro and in vivo. Proc Natl Acad Sci U S A 97: 13625- 13630.

Gronthos, S, Brahim, J, Li, W et al ( 2002). Stem cell properties of human dental pulp stem cells. J Dent Res 81: 531- 535.

Gronthos, S, Zannettino, AC, Hay, SJ et al ( 2003). Molecular and cellular characterisation of highly purified stromal stem cells derived from human bone marrow. J Cell Sci 116: 1827- 1835.

Hasegawa, M, Yamato, M, Kikuchi, A, Okano, T, Ishikawa, I ( 2005). Human periodontal ligament cell sheets can regenerate periodontal ligament tissue in an athymic rat model. Tissue Eng 11: $469-477$.

Ivanovski, S, Haase, HR, Bartold, PM ( 2001). Isolation and characterization of fibroblasts derived from regenerating human periodontal defects. Arch Oral Biol 46: 679-688.

Karring, T, Nyman, S, Lindhe, J ( 1980). Healing following implantation of periodontitis affected roots into bone tissue. J Clin Periodontol 7: 96- 105.

Keller, GM. ( 1995). In vitro differentiation of embryonic stem cells. Curr Opin Cell Biol 7: 862-869.

Kemp, KC, Hows, J, Donaldson, C ( 2005). Bone marrow-derived mesenchymal stem cells. Leuk Lymphoma 46: 1531- 1544.

Kramer, PR, Nares, S, Kramer, SF, Grogan, D, Kaiser, M ( 2004). Mesenchymal stem cells acquire characteristics of cells in the periodontal ligament in vitro. J Dent Res 83: 27-34.

Limeback, H, Sodek, T, Aubin, JE ( 1983). Variation in collagen expression by cloned periodontal ligament cells. J Periodontal Res 18: 242- 248.

Majumdar, MK, Keane-Moore, M, Buyaner, D et al ( 2003). Characterization and functionality of cell surface molecules on human mesenchymal stem cells. J Biomed Sci 10: 228- 241.

Mankani, MH, Kuznetsov, SA, Fowler, B, Kingman, A, Robey, PG ( 2001). In vivo bone formation by human bone marrow stromal cells: effect of carrier particle size and shape. Biotechnol Bioeng 72: 96- 107. 
McCulloch, CAG ( 1985). Progenitor cell populations in the periodontal ligament of mice. Anat Rec 211: 258-262.

McCulloch, CAG, Melcher, AH ( 1983a). Cell density and cell generation in the periodontal ligament of mice. Am J Anat 167: 43- 58.

McCulloch, CAG, Melcher, AH ( 1983b). Cell migration in the periodontal ligament of mice. J Periodontal Res 18: 339- 352.

McCulloch, CAG, Melcher, AH ( 1983c). Continuous labelling of the periodontal ligament of mice. J Periodontal Res 18: 231- 241.

McCulloch, CAG, Nemeth, E, Lowenburg, B, Melcher, AH ( 1987). Paravascular cells in endosteal spaces of alveolar bone contribute to periodontal ligament cell populations. Anat Rec 219: 2233- 2242.

McCulloch, CAG, Barghava, U, Melcher, AH ( 1989). Cell death and regulation of populations of cells in the periodontal ligament. Cell Tissue Res 255: 129- 138.

Mezey, E, Key, S, Vogelsang, G, Szalayova, I, Lange, GD, Crain, B ( 2003). Transplanted bone marrow generates new neurons in human brains. Proc Natl Acad Sci U S A 100: 1364- 1369.

Miura, M, Gronthos, S, Zhao, M et al ( 2003). SHED: stem cells from human exfoliated deciduous teeth. Proc Natl Acad Sci U S A 100: 5807- 5812.

Morsczeck, C, Gotz, W, Schierholz, J et al ( 2005). Isolation of precursor cells (PC) from human dental follicle of wisdom teeth. Matrix Biol 24: 155- 165.

Murphy, JM, Fink, DJ, Hunziker, EB, Barry, FP ( 2003). Stem cell therapy in a caprine model of osteoarthritis. Arthritis Rheum 48: 3464- 3474.

Nemeth, E, Kulkarnie, G, McCulloch, CAG ( 1993). Disturbances of gingival fibroblast population homeostasis due to experimentally induced inflammation in the cynomolgus monkey (Macaca fascicularis): potential mechanism for disease progression. J Periodontal Res 28: 180- 190.

Nyman, S, Karring, T, Lindhe, J, Planten, S ( 1980). Healing following implantation of periodontitisaffected roots into gingival connective tissue. J Clin Periodontol 7: 394- 401.

Ohazama, A, Modino, SA, Miletich, I, Sharpe, PT ( 2004). Stem-cell-based tissue engineering of murine teeth. J Dent Res 83: 518- 522.

Palmer, RM, Lumsden, AG ( 1987). Development of periodontal ligament and alveolar bone in homografted recombinations of enamel organs and papillary, pulpal and follicular mesenchyme in mouse. Arch Oral Biol 32: 281- 289.

Pender, N, Heaney, TG, Pycock, D, West, CR ( 1988). Progenitor connective tissue cell populations in the gingival papillae of the rat. J Periodontal Res 23: 175- 181.

Seo, B, Miura, M, Gronthos, S et al ( 2004). Investigation of multipotent postnatal stem cells from human periodontal ligament. Lancet 364: 149- 155.

Seo, B, Miura, M, Sonoyama, W, Coppe, C, Stanyon, R, Shi, S ( 2005). Recovery of stem cells from cryopreserved periodontal ligament. J Dent Res 84: 907-912. 
Shi, S, Gronthos, S (2003). Perivascular niche of postnatal mesenchymal stem cells in human bone marrow and pulp. J Bone Miner Res 18: 696- 704.

Shih, IM ( 1999). The role of CD146 (Mel-CAM) in biology and pathology. J Pathol 189: 4- 11.

Simmons, PJ, Torok-Storb, B ( 1991). Identification of stromal cell precursors in human bone marrow by a novel monoclonal antibody, STRO-1. Blood 78: 55-62.

Stamm, C, Westphal, B, Kleine, HD et al ( 2003). Autologous bone-marrow stem-cell transplantation for myocardial regeneration. Lancet 361: 45- 46.

Ten Cate, AR, Mills, C ( 1972). The development of periodontium: the origin of alveolar bone. Anat Rec 173: 69- 72.

Ten Cate, AR, Mills, C, Solomon, G ( 1971). The development of the periodontium. A transplantation and autoradiographic study. Anat Rec 173: 365- 374.

Trubiani, O, Di Primio, R, Triani, T et al ( 2005). Morphological and cytoflurometric analysis of adult mesenchymal stem cells expanded ex vivo from periodontal ligament. Int J Immunopathol Pharmacol 18: 213- 221.

Wang, HL, Greenwell, H, Fiorellini, J et al ( 2005). Research, Science and Therapy Committee. Periodontal regeneration. J Periodontol 76: 1601- 1622.

Zohar, R, Tenenbaum, HC. ( 2005). How predictable are periodontal regenerative procedures? J Can Dent Assoc 71: 675-680.

\section{Author Affiliations}

S. Ivanovski, School of Dentistry, University of Queensland, Brisbane, Qld

S. Gronthos, Mesenchymal Stem Cell Group, Division of Haematology, Institute of Medical and Veterinary Science, Adelaide, SA, Australia

S. Shi, National Institute of Dental and Craniofacial Research, National Institutes of Health, Bethesda, MD, USA

P. M. Bartold, Colgate Australian Clinical Dental Research Centre, Dental School, University of Adelaide, Adelaide, SA, Australia 